\title{
Isospin emission and flow at high baryon density: a test of the symmetry potential
}

\author{
V. Giordano ${ }^{1,2}$, M. Colonna ${ }^{1}$, M. Di Toro ${ }^{1,2, *}$, V.Greco ${ }^{1,2}$, J. Rizzo ${ }^{1,2}$ \\ 1 Laboratori Nazionali del Sud, INFN, \\ Via S. Sofia 62, I-95123 Catania \\ 2 Physics and Astronomy Dept., \\ University of Catania, Italy \\ * email: ditoro@lns.infn.it
}

\begin{abstract}
High energy Heavy Ion Collisions (HIC) are studied in order to access nuclear matter properties at high density. Particular attention is paid to the selection of observables sensitive to the poorly known symmetry energy at high baryon density, of large fundamental interest, even for the astrophysics implications. Using fully consistent transport simulations built on effective theories we test isospin observables ranging from nucleon/cluster emissions to collective flows (in particular the elliptic, squeeze out, part). The effects of the competition between stiffness and momentum dependence of the Symmetry Potential on the reaction dynamics are thoroughly analyzed. In this way we try to shed light on the controversial neutron/proton effective mass splitting at high baryon and isospin densities. New, more exclusive, experiments are suggested.
\end{abstract}

PACS numbers: 21.30.Fe, 21.65.-f, 25.75.Dw, 25.75.Ld

Keywords: High Energy Heavy Ion Collisions, Fast Nucleon/Cluster Emission, Isospin Collective Flows, Symmetry Energy at High Baryon Density, Symmetry Potentials, Neutron/Proton Effective Masses

\section{INTRODUCTION}

In the nuclear Equation of State $(E o S)$ the symmetry energy $E_{\text {sym }}$ appears in the energy density $\epsilon\left(\rho, \rho_{3}\right) \equiv$ $\epsilon(\rho)+\rho E_{\text {sym }}\left(\rho_{3} / \rho\right)^{2}+O\left(\rho_{3} / \rho\right)^{4}+. .$, expressed in terms of total $\left(\rho=\rho_{p}+\rho_{n}\right)$ and isospin $\left(\rho_{3}=\rho_{p}-\rho_{n}\right)$ densities. The symmetry term gets a kinetic contribution directly from basic Pauli correlations and a potential part from the highly controversial isospin dependence of the effective interactions [1-3]. Both at sub-saturation and supra-saturation densities, predictions based on the existing many-body techniques diverge rather widely, see [4] . We remind that the knowledge of the $E o S$ of asymmetric matter is very important at low densities (neutron skins, nuclear structure at the drip lines, neutron distillation in fragmentation, neutron star formation and crust..) as well as at high densities (transition to a deconfined phase, neutron star mass/radius, cooling, hybrid structure, formation of black holes...). We take advantage of recent opportunities in theory (development of rather reliable microscopic transport codes for $H I C$ ) and in experiments (availability of very asymmetric radioactive beams, improved possibility of measuring event-by-event correlations) to present results that could severely constrain the existing effective interaction models. We will discuss dissipative collisions at intermediate energies, i.e. in the range from $100 \mathrm{AMeV}$ up to $1 \mathrm{AGeV}$. This will allow to probe the symmetry term above normal density.

The reaction is simulated using transport codes based on effective mean field theories, with correlations/fluctuations included via hard nucleon-nucleon collisions, see [1, 5, 6]. The dynamical effects of a different density dependence of the isovector part of the $E o S$ are tested [7]. Moreover a particular attention is devoted to the isospin effects on the momentum dependence of the symmetry potentials, i.e. to observables sensitive to a different neutron/proton effective mass in asymmetric matter. The problem of Momentum Dependence in the Isovector channel $(I s o-M D)$ is still very controversial and it would be extremely important to get more definite experimental information, see the refs. 1, 2, 8]. Exotic beams at intermediate energies are of interest in order to have high momentum particles and to test regions of high baryon (isoscalar) and isospin (isovector) density during the reaction dynamics.

The paper is arranged as follows. In Sect.2 the selfconsistent transport approach to the reaction dynamics is shortly described. Sect. 3 is devoted to a detailed analysis of the used effective interactions, in particular of the isovector contributions to the local and non-local part of the mean field potentials. The latter, which corresponds to the $I s o-M D$ case, leads to a splitting of the neutron-proton effective masses in asymmetric matter. Results for ${ }^{197} A u+{ }^{197} A u$ are shown in Sect.4, selecting the observables that would allow an independent study of the two isovector contributions. Conclusions and perspectives are presented in Sect.5.

\section{THE COLLISION DYNAMICS}

We perform ab initio collision simulations using the microscopic Stochastic Mean Field (SMF) model. It is based on mean field transport theory with correlations included via hard nucleon-nucleon (NN) collisions and with inclusion of stochastic forces acting on the mean phase-space trajectory [1, 5, ,9 11]. Stochasticity is essential in order to allow the growth of dynamical instabilities leading to fragment production, as well as to obtain physical widths of the observable distributions. Moreover it will allow to perform event-by-event correlation studies of great importance for the very complex reaction dynamics 
in this energy range.

The transport equation for the phase space distribution function, with the Pauli blocking consistently evaluated, is integrated following a representation in terms of test particles of finite widths [9, 10]. A detailed description of the procedure is given in ref. [1]. Our code [12] has been extended by the introduction of momentum dependent mean fields (see next Section), which are rather important in this energy range. It has been also possible to improve the numerical accuracy while even reducing the computing times [13].

A parametrization of free nucleon-nucleon cross sections is used, with isospin, energy and angular dependence [14]. Low energy NN collisions, mostly forbidden because of the Pauli blocking, have large cross sections and could induce spurious effects in the presence of some numerical inefficiency in the blocking procedure, due to the discretization of the phase space. In order to avoid such problems a cutoff value $\sigma_{c u t}=50 \mathrm{mb}$ is used in our calculations. A parallel ensemble method is employed in the implementation of the collision term.

For discussions of isospin dynamics in this energy regime it is essential to have a reliable procedure for fragment recognition, i.e. to identify free and clusterized nucleons. A coalescence procedure is applied in an event by event analysis at the "freeze-out" time, i.e. when the resulting fragments are well separated in space and interacting only via Coulomb forces. The fragments are formed using a phase-space proximity criterion. Two particles are recognized to belong to the same cluster if they are sufficiently close in phase space. The procedure is applied to several random samplings of $\mathrm{N}$ (total number of nucleons) test particles from the full ensemble distribution at the freeze-out. The used coalescence parameters are $d_{r}=4.5 \mathrm{fm}, d_{p}=1.5 \mathrm{fm}^{-1}$, that reasonably reproduce the charge distributions in the same energy range [15]. We have also seen that the results do not show appreciable changes for small variations of the coalescence parameters. Since the random sampling usually is not exactly preserving energy and momentum conservation, we apply an additional constraint to impose them.

\section{MOMENTUM DEPENDENCE OF THE FIELDS}

We adopt a generalized form of the effective interactions, which can be easily reduced to Skyrme-like forces, with momentum dependent terms also in the isovector channel [8, 13, 16, 17]. The general structure of the isoscalar and isovector Momentum Dependent (MD) effective fields is derived via an isospin asymmetric extension of the Gale-Bertsch-DasGupta (GBD) force [18 22], which corresponds to a Yukawian non-locality.

The energy density is parametrized as follows:

$$
\left.\varepsilon(\vec{r})=\varepsilon_{k i n}(\vec{r})+\varepsilon_{A}(\varrho(\vec{r}))+\varepsilon_{B}(\varrho(\vec{r}))+\varepsilon_{C, z}(\vec{r})\right)
$$

The kinetic term is:

$$
\varepsilon_{k i n}(\vec{r})=\int \frac{d^{3} p}{h^{3}}\left[f_{n}(\vec{r}, \vec{p})+f_{p}(\vec{r}, \vec{p})\right] \frac{p^{2}}{2 m}
$$

The terms $\varepsilon_{A}$ and $\varepsilon_{B}$ of Eq.(1) account for saturation properties, including the symmetry energy,

$$
\begin{gathered}
\varepsilon_{A}(\vec{r})=\frac{A}{2} \frac{\varrho^{2}}{\varrho_{0}}-\frac{A}{3}\left(\frac{1}{2}+x_{0}\right) \frac{\varrho^{2}}{\varrho_{0}} \beta^{2} \\
\varepsilon_{B}(\vec{r})=\frac{B}{\sigma+1} \frac{\varrho^{\sigma+1}}{\varrho_{0}^{\sigma}}-\frac{2}{3} \frac{B}{\sigma+1}\left(\frac{1}{2}+x_{3}\right) \frac{\varrho^{\sigma+1}}{\varrho_{0}^{\sigma}} \beta^{2}
\end{gathered}
$$

where $\rho$ is the "local" density, $\rho_{0}$ the saturation value and $\beta=(N-Z) /(N+Z)$ represents the "local" asymmetry parameter. The last term gives the momentum dependence and can be written as [17]:

$$
\varepsilon_{C, z}(\vec{r})=\frac{8(C+2 z)}{5 \varrho_{0}} I_{n p}(\vec{r})+\frac{4(3 C-4 z)}{5 \varrho_{0}}\left(I_{n n}(\vec{r})+I_{p p}(\vec{r})\right)
$$

The momentum dependence is contained in the $\mathcal{I}_{\tau \tau^{\prime}}$ terms, which are integrals of the form

$$
\mathcal{I}_{\tau \tau^{\prime}}=\int d \vec{p} d \vec{p}^{\prime} f_{\tau}(\vec{r}, \vec{p}) f_{\tau^{\prime}}\left(\vec{r}, \vec{p}^{\prime}\right) g\left(\vec{p}, \vec{p}^{\prime}\right),
$$

with $\tau=P, N$, for protons and neutrons. Here $f_{\tau}(\vec{r}, \vec{p})$ are the nucleon phase space distributions for protons and neutrons and the function $g\left(\vec{p}, \vec{p}^{\prime}\right) \equiv g\left[\left(\vec{p}-\vec{p}^{\prime}\right)^{2}\right]$ determines the type of momentum dependence. A Skyrme-like momentum dependence is obtained when we use the simple quadratic form $g\left(\vec{p}, \vec{p}^{\prime}\right)=\left(\vec{p}-\vec{p}^{\prime}\right)^{2}$. A more general momentum dependence, in better agreement with phenomenological optical potentials, can be introduced by the function [18 22]

$$
g\left(\vec{p}, \vec{p}^{\prime}\right)=\left[1+\left(\frac{\vec{p}-\vec{p}^{\prime}}{\Lambda}\right)^{2}\right]^{-1} .
$$

For symmetric nuclear matter $(\beta=0)$ the energy density Eqs. (21314/5) reduces to the parametrization proposed by Gale, Bertsch and Das Gupta [18, 19], that for low densities and momenta gives the same results of the Skyrme-Gogny force, see 21].

The EoS of symmetric matter is fixed by the parameters $\mathrm{A}, \mathrm{B}, \mathrm{C}, \sigma$ and $\Lambda$. We choose the values $A=-111.3 \mathrm{MeV}, B=141.3 \mathrm{MeV}, C=-64.5 \mathrm{MeV}$, $\sigma=7 / 6$ and $\Lambda=1.5 p_{F}^{0}$ (with $p_{F}^{0}$ Fermi momentum at normal density), which provide a rather Soft EoS with compressibility $K \simeq 215 \mathrm{MeV}$ and an isoscalar effective mass $m^{*} / m=0.67$ at saturation density $\rho_{0}=0.16 \mathrm{fm}^{-3}$. We note that an indication for a Soft Eos (Isoscalar) even at higher densities comes also from heavy ion data (collective flows and meson production) at intermediate energies 23]. 


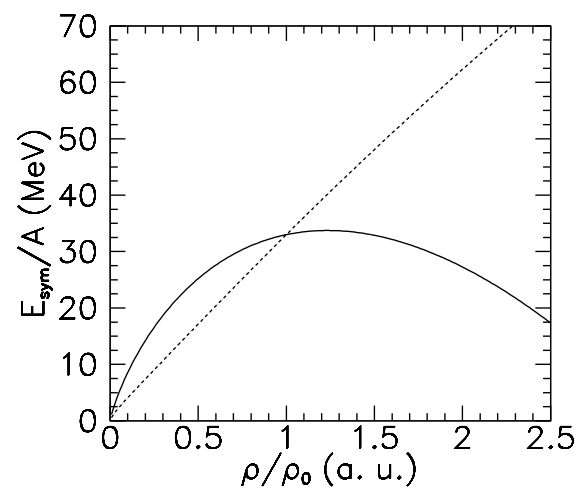

FIG. 1: Density dependence of the symmetry energies used in the simulations presented here: asy-soft (solid) and asy-stiff (dashed).

From the energy density one derives the mean field potentials as $U_{\tau}(\vec{r}, \vec{p})=\delta \varepsilon / \delta f_{\tau}$. Thus the above energy density implies a momentum dependent mean field interaction. The momentum dependence is isoscalar if, in the Eq.(5), the coefficients in front of $I_{n p}(\vec{r})$ and in front of the combination $\left(I_{n n}(\vec{r})+I_{p p}(\vec{r})\right)$ are equal (corresponding to the $C=8 z$ case), but it can also get an isovector part, if they are different.

The isovector momentum dependence implies different effective masses for protons and neutrons given as $\frac{m_{\tau}^{*}}{m}=$ $\left(1+\frac{m}{\hbar^{2} p} \frac{\partial U_{\tau}}{\partial p}\right)^{-1}$, for $p=p_{F, \tau}$, at fixed density.
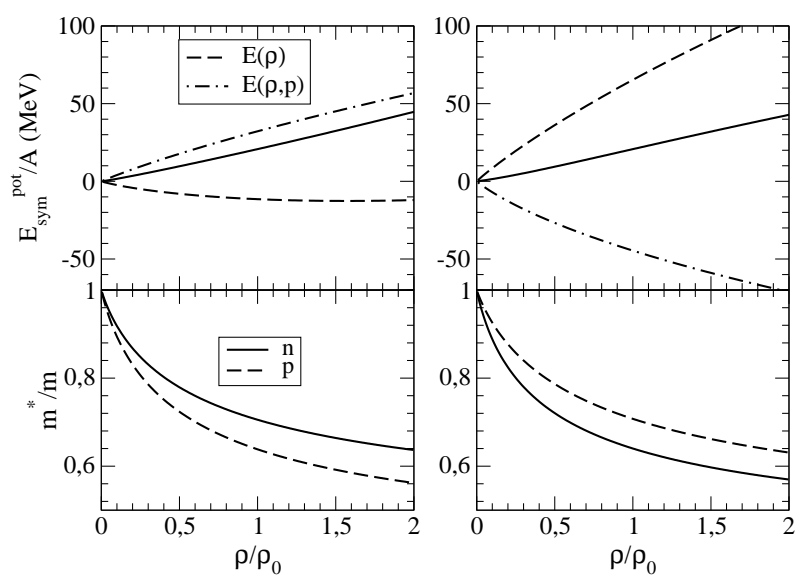

FIG. 2: Upper Panels: Density dependence of the potential symmetry energy (Solid Lines), in the Asystiff choice. Dashed lines refer to local contributions, dot-dash lines to momentum-dependent ones, see text. Left: $m_{n}^{*}>m_{p}^{*}$ parametrization; Right: $m_{n}^{*}<m_{p}^{*}$ case. Lower Panels: corresponding behavior of neutron/proton effective masses as a function of the density, for an asymmetry $\beta=0.2$.

Here we probe the sensitivity of isospin observables to two essentially different density dependence of the symmetry energy around saturation: Asy-soft with a smooth behavior below saturation, and even a decrease at high densities, vs. Asy-stiff which always shows instead a rapid increase, roughly proportional to the density $[1,[3,7]$. In fact the two choices are well characterized by the slope parameter

$$
L \equiv 3 \rho_{0}\left(d E_{\text {sym }} / d \rho\right)_{\rho=\rho_{0}}
$$

which is of the order of $10-20 \mathrm{MeV}$ in the Asysoft case, just from the kinetic contribution to the symmetry term, and around $70-100 \mathrm{MeV}$ in the Asystiff parametrization with also a repulsive potential part [1, 7]. In Fig.1 we show the density dependence for these two typical choices.

Table 1. Parameters $x_{0}, x_{3}, z$ for opposite mass splittings, with the same $E_{\text {sym }}=33 \mathrm{MeV}$, for Asysoft $(\mathrm{L}=19 \mathrm{MeV})$ and Asystiff $(\mathrm{L}=95 \mathrm{MeV})$.

\begin{tabular}{c|c|c|c|}
\hline Asysoft & & & \\
\hline Mass splitting & $x_{0}$ & $x_{3}$ & $z(\mathrm{MeV})$ \\
\hline$m_{n}^{*}>m_{p}^{*}$ & 1.111 & 1.196 & -35.467 \\
$m_{n}^{*}<m_{p}^{*}$ & 5.212 & 2.988 & 44.971 \\
$m_{n}^{*}=m_{p}^{*}$ & 3.165 & 2.094 & 4.811 \\
\hline Asystiff & & & \\
\hline Mass splitting & $x_{0}$ & $x_{3}$ & $z$ \\
\hline$m_{n}^{*}>m_{p}^{*}$ & -1.614 & -1.210 & -35.467 \\
$m_{n}^{*}<m_{p}^{*}$ & 2.487 & 0.582 & 44.971 \\
$m_{n}^{*}=m_{p}^{*}$ & 0.440 & 0.312 & 4.811 \\
\hline
\end{tabular}

When we use momentum-dependent interactions we have also contributions to the symmetry energy from the non-local terms. The presence of an interplay between the $C$ and $z$ parameters in our form of the effective interaction allows an independent study of the dynamical effects of the stiffness of the symmetry term and of the neutron/proton effective mass splitting. This can be easily done just varying the $x_{0}, x_{3}$ and $z$ parameters. In Table I we report the used set of parameters. The symmetry energy at saturation is always fixed to $E_{\text {sym }}\left(\rho_{0}\right)=33 \mathrm{MeV}$.

In Fig 2 we plot the density dependence of the potential part of the symmetry energy, in the Asystiff case, for the two choices of the $\mathrm{n} / \mathrm{p}$ mass splitting (solid lines, upper panels). We also separately report the contributions from the momentum-dependent, $E(\rho, p)$, and the density dependent, $E(\rho)$, part of the EoS, whose sum gives the total $E_{s y m}^{p o t}$. A change in the sign of the mass splitting is related to opposite behaviors of these two contributions, exactly like it happens in Skyrme-like forces, see sections (2.1-2.2) of ref. [1]. The lower panels show the density dependence of the corresponding mass splitting, for an asymmetry parameter $\beta=0.2$ (the ${ }^{197} \mathrm{Au}$ asymmetry). In order to probe the mass splitting effects on the heavy ion dynamics we have chosen parametrizations that give almost opposite splittings at all densities.

In Fig 3 we present the density dependence of the neutron/proton symmetry potentials, for the two stiffness of 


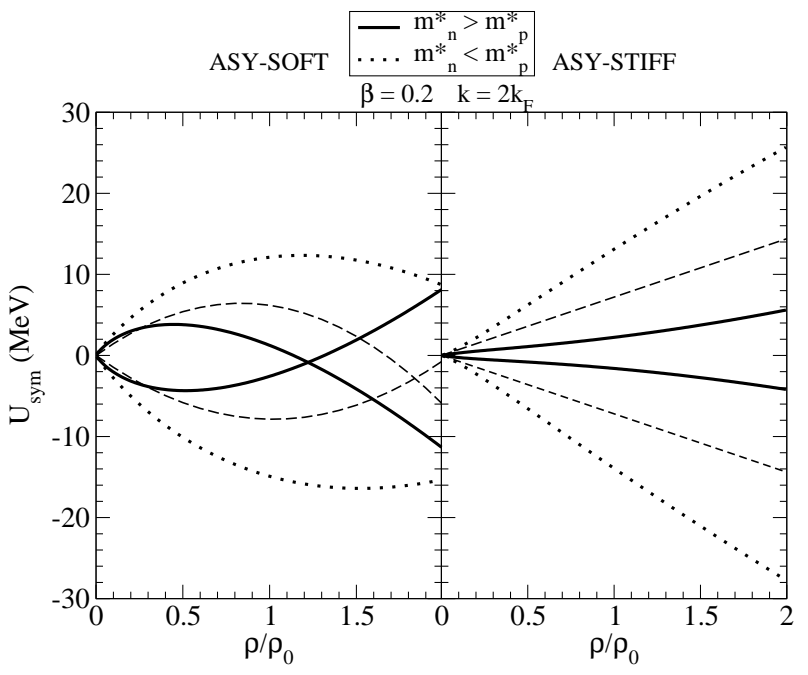

FIG. 3: Density dependence of neutron(upper)-proton(lower) potentials for an asymmetry $\beta=0.2$ for the Asysoft(left) and Asystiff (right) choices. Dashed: No momentum dependence. Momentum dependent potentials at $k=2 k_{F}$ : solid lines for the $m_{n}^{*}>m_{p}^{*}$ case, dotted lines for the opposite $m_{n}^{*}<m_{p}^{*}$ choice.

the symmetry term, evaluated in the case without momentum dependence (dashed lines) and in the momentum dependence $(I s o-M D)$ case for the $m_{n}^{*}<m_{p}^{*}$ (dotted) and the opposite $m_{n}^{*}>m_{p}^{*}$ (solid) choices. We see that the momentum dependence modifies the effect of the symmetry term stiffness on the nucleon potentials, with differences that become more appreciable with increasing nucleon momenta. From this figure we can already predict large effects of the effective mass splitting at high momenta.

This is shown more explicitly in Fig 4 where we see the momentum dependence of the neutron-proton potentials at saturation density for the two mass splitting choices, always for a "typical" $\beta=0.2$ asymmetry $\left({ }^{124} S n,{ }^{197} A u \ldots\right)$. The plot is for the Asysoft (left panel) and the Asystiff (right) symmetry term, and in fact it is not much different. Indeed we can see also from the previous Fig 3 that at normal density the difference between neutron and proton potentials is almost the same for the two asy-stiffness, even in the case of $I s o-M D$ interactions..

The Figs 3 and 4 suggest the presence of interesting Isoscalar and Isovector $M D$ effects on the reaction dynamics:

- Isoscalar. In general the momentum dependence gives more attractive potentials at low momenta, $p<p_{F}$, and more repulsive at high $p, p>p_{F}$. In the reaction dynamics we expect the more energetic nucleons to be fast emitted and to suffer less collisions [18]. As a consequence we will have less stopping of the matter and less compression. The isoscalar EoS becomes stiffer when the momentum dependence is included.

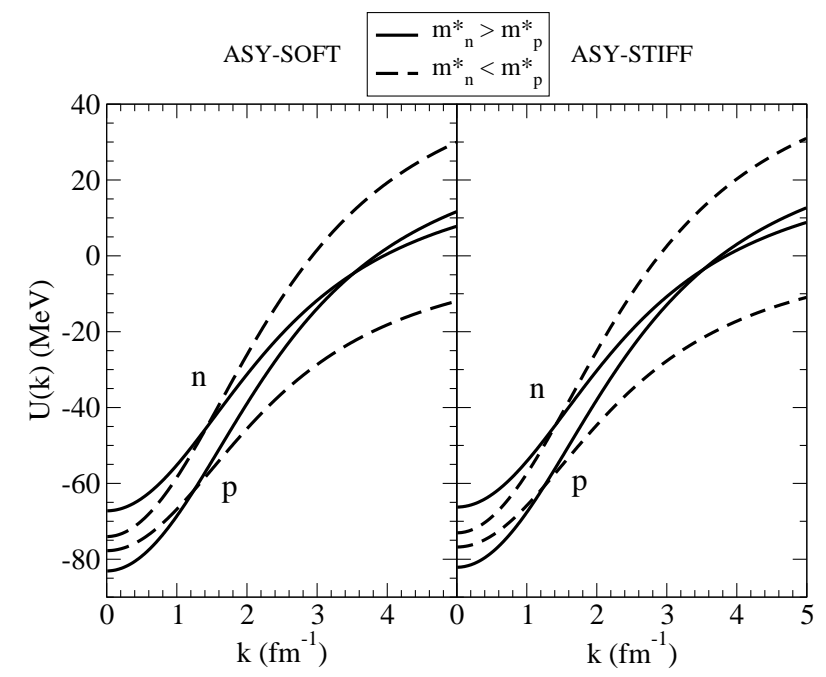

FIG. 4: Momentum dependence of neutron-proton potentials at saturation density and asymmetry $\beta=0.2$, for the two splitting choices $m_{n}^{*}<m_{p}^{*}$ (dashed) and $m_{n}^{*}>m_{p}^{*}$ (solid). Left panel: Asysoft Iso-Eos. Right panel: Asystiff case

- Isovector. Isospin effects on the momentum dependence imply different slopes around $p_{F}$ for neutrons and protons, as clearly shown in Fig 4 , and so the larger repulsion above $p_{F}$ is different. In the case $m_{n}^{*}<m_{p}^{*}$ the high momentum neutrons will see a more repulsive field with respect to the high- $p$ protons. The opposite will happen in the $m_{n}^{*}>m_{p}^{*}$ case. The fast nucleon emission will be directly affected: in the $m_{n}^{*}<m_{p}^{*}$ case we expect a larger $n / p$ yield for nucleons emitted in central collisions and a larger neutron Squeeze-out (elliptic flow) in semicentral collisions in heavy ion reactions at intermediate energies, in particular for high $p_{t}$ (transverse momentum) selections. In fact in the interacting, high density, early stage of the reaction dynamics the pressure is built from violent nucleon-nucleon collisions and the high $p_{t}$ particles will carry the maximal information on high density and momentum dependence of the symmetry potentials. The azimuthal distributions (elliptic flows) will be particularly affected since particles mostly retain their high transverse momenta escaping along directions orthogonal to the reaction plane without suffering much rescattering processes.

We will test those predictions also for n-rich vs. npoor light ions, like $\left({ }^{3} \mathrm{H},{ }^{3} \mathrm{He}\right)$, easier to detect. Since, as already noted, the symmetry potentials are not very different in the Asystiff/Asysoft choice for density range probed at intermediate energies (see the discussion in the next Section), we can expect that the Mass - Splitting effect could be even larger than the one related to the different stiffness of the symmetry term.

A final interesting point is about the crossing of 

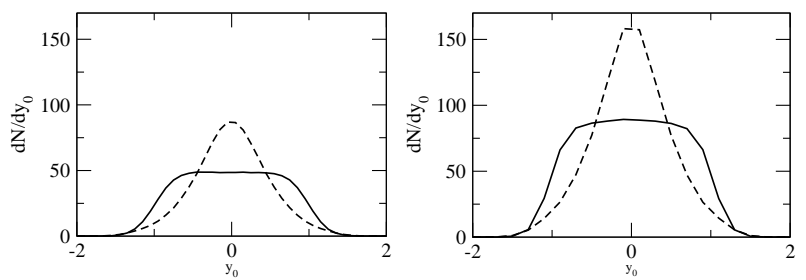

FIG. 5: $197 \mathrm{Au}+197 \mathrm{Au}$ at $400 \mathrm{AMeV}$ (left) and $600 \mathrm{AMeV}$ (right), central collision. Longitudinal (solid lines) and Transversal (dashed lines) scaled rapidity distributions of $\mathrm{Z}=1-3$ ions.

the two predictions, with opposite mass-splitting choice, at high momenta, see Fig,4. Roughly this should happen around the Fermi momentum corresponding to the density reached in the compressed source of the fast emitted nucleons. This represents an independent way to check the maximum density reached during the collision.

\section{RESULTS ON ${ }^{197} A u+{ }^{197} A u$ REACTIONS AT INTERMEDIATE ENERGIES}

Our Stochastic Mean Field $(S M F)$ transport code has been implemented with $I s o-M D$ symmetry potentials, with a different $(n, p)$ momentum dependence, as discussed in detail in the previous section. This will allow to follow the dynamical effect of opposite $n / p$ effective mass splitting while keeping the same density dependence of the symmetry energy [17].

We present here some results for ${ }^{197} A u+{ }^{197} A u$ reactions at $400 \mathrm{AMeV}$ and $600 \mathrm{AMeV}$ 24]. For central collisions in the interacting zone we can reach baryon densities about $1.7-1.8 \rho_{0}$ in a transient time of the order of $15-20 \mathrm{fm} / \mathrm{c}$. The system is quickly expanding and the Freeze-Out time is around $50 \mathrm{fm} / \mathrm{c}$. At this time we have a dominant Coulomb interaction among the reaction products. All the results presented here refer to this time step. Secondary decays of excited primary fragments are not accounted for. In fact this will not affect too much the properties of nucleons and light ions at high transverse momenta mostly discussed in this work.

For each beam energy and centrality we run 50 independent events. In order to show how global properties of the reaction are reproduced by the simulations, in Fig 5 we report the longitudinal and transversal rapidity distributions of protons and light ions $(\mathrm{Z}=1-3)$ in central collisions at the two beam energies. We use the $C M$ scaled rapidity $y_{0} \equiv y_{c m} / y_{\text {proj }}$, vs. the projectile rapidity. A good parameter to evaluate the stopping power of the collision is the $V$ artl quantity, i.e. the ratio between the variances of the transverse and longitudinal rapidity distributions, recently suggested by the FOPI collaboration, $[25,26]$. In our calculation we get values around 0.75 , not much different at the two beam energies, comparable to data although no experimental trigger is included. We
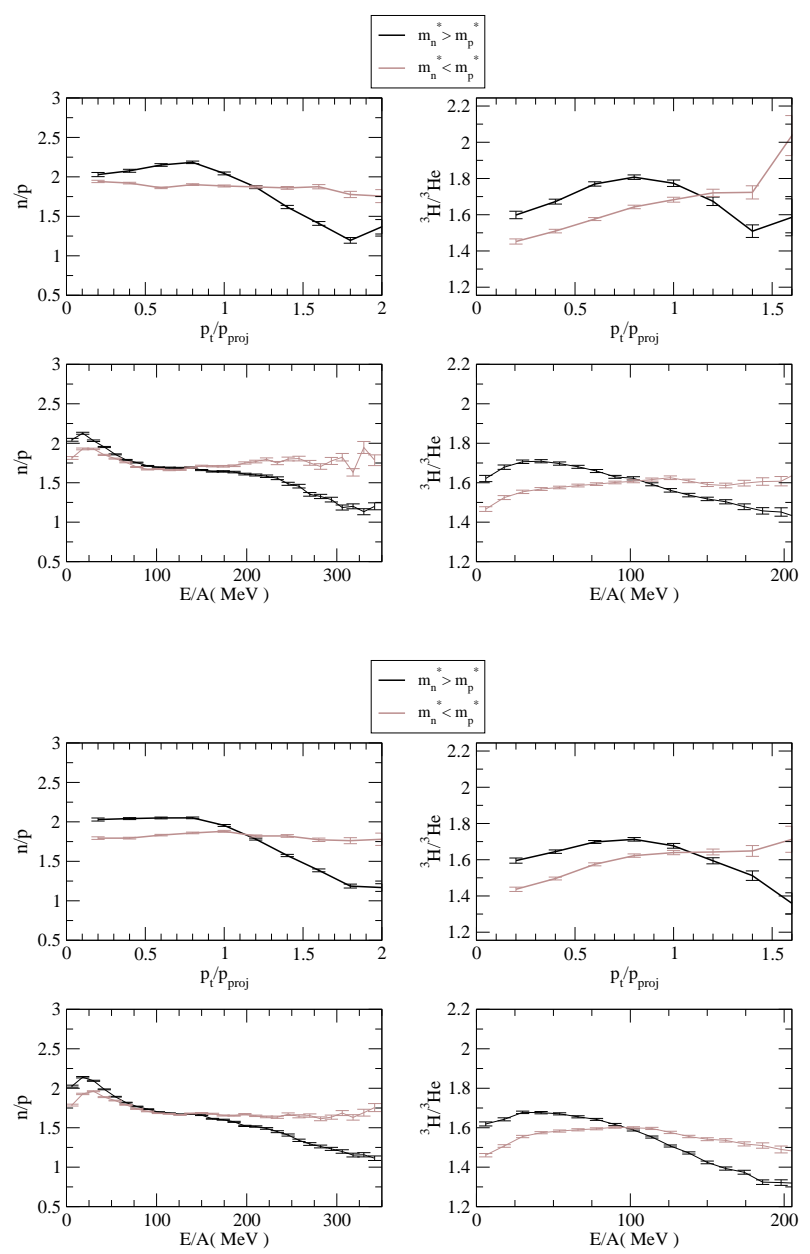

FIG. 6: $197 \mathrm{Au}+197 \mathrm{Au}$ at $400 \mathrm{AMeV}$, central collision.Isospin content of nucleon (left) and light ion (right) emissions vs. $p_{t}$ at midrapidity, $\left|y_{0}\right|<0.3$, (upper) and kinetic energy (lower), for all rapidities, for the two nucleon mass splitting choices. Top Panels: Asysoft; Bottom Panels: Asystiff.

note that this estimation of the stopping power appears not dependent on the isovector contributions to the EoS. This clearly shows the difficulty of the search for well measurable isospin effects in the reaction dynamics. Here we will focus our attention on the isospin content of fast emitted nucleons and light ions and on isospin collective flows.

\section{Isospin ratios of fast emitted particles}

In Figs 6.77 we present the $(n / p)$ and ${ }^{3} \mathrm{H} /{ }^{3} \mathrm{He}$ yield ratios at freeze-out, for two choices of Asy-stiffness and Mass-splitting, vs. transverse momentum in a midrapidity selection (upper curves) and kinetic energy ( all rapidities, lower curves). In this way we can separate particle emissions from sources at different densities, as discussed in the previous Section.

For both beam energies we clearly observe the opposite 

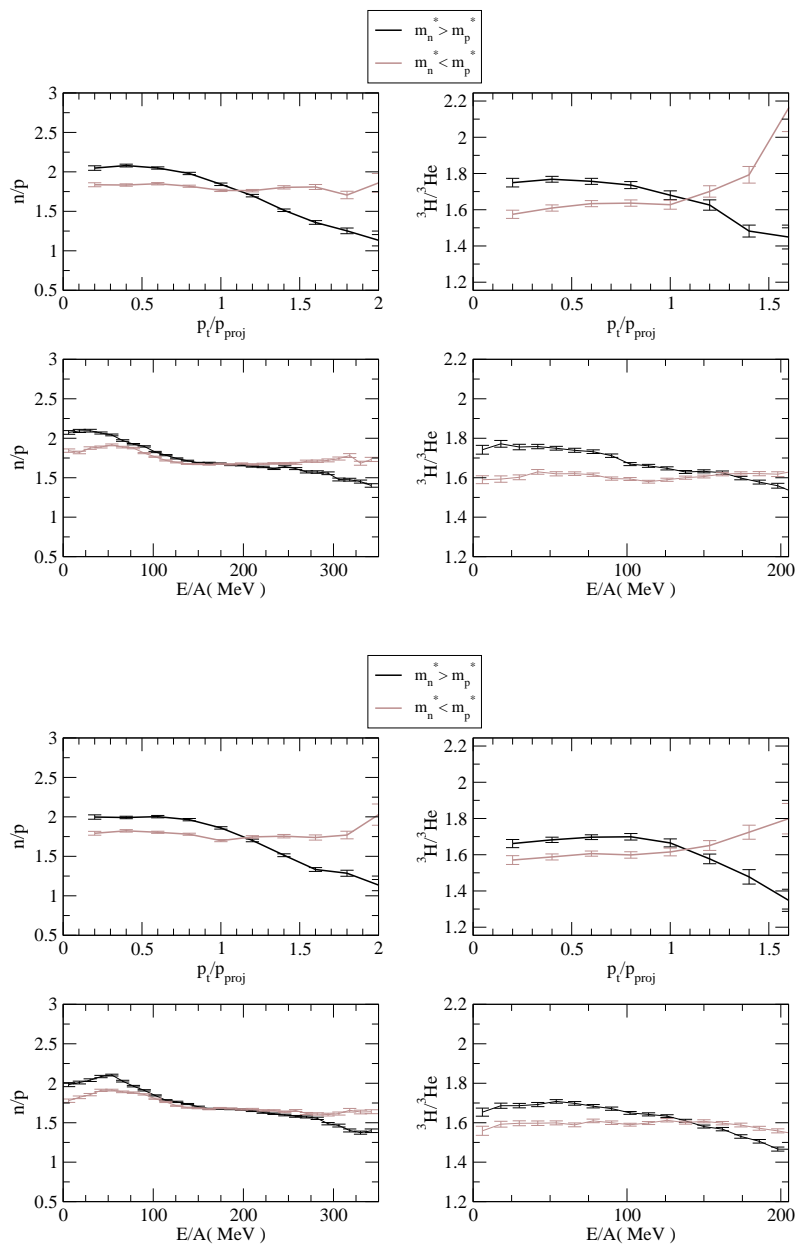

FIG. 7: Same as in Fig.(6) for $197 \mathrm{Au}+197 \mathrm{Au}$ at $600 \mathrm{AMeV}$, central collision.

effect of the different mass splitting in the low and high momentum regions, as expected from Fig 4 E.g. in the $m_{n}^{*}<m_{p}^{*}$ case the neutrons see a less repulsive potential at low momenta and a more repulsive one at high $p_{t}$. The curves in the opposite mass-splitting show exactly the opposite behavior. We note some interesting features:

i) The effect is almost not dependent on the stiffness of the symmetry term. At high $p_{t}$, where particles mostly come from high density regions, the larger repulsion seen by neutrons in the Asy-stiff case, leading to an enhanced emission, is compensated by the larger Coulomb repulsion in the remaining matter, favoring proton emission. On the other hand, at low $p_{t}$, the sensitivity to the Asystiffness is lost due to the mixing of sources at different densities, also for central rapidities, during the radial expansion.

ii) The curves are crossing at $p_{t} \simeq p_{\text {projectile }}=$ $2.13 \mathrm{fm}^{-1}$. The crossing nicely corresponds to the Fermi momentum of a source at baryon density $\rho \simeq 1.6 \rho_{0}$, [8, 16, 17].

iii) The results appear not very sensitive to the beam energy going from 400 to $600 \mathrm{AMeV}$, likely because the
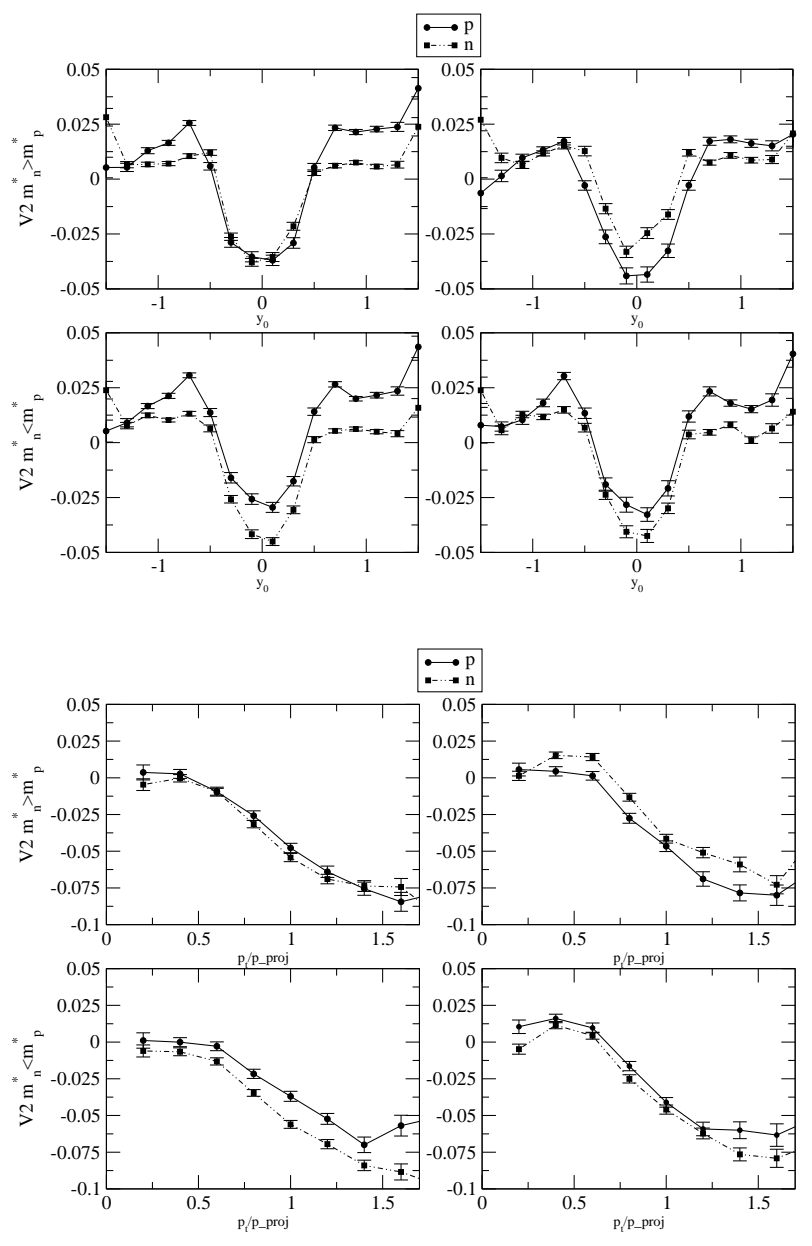

FIG. 8: Proton (thick) and neutron (thin) $V_{2}$ flows in a semicentral reaction $\mathrm{Au}+\mathrm{Au}$ at $400 \mathrm{AMeV}$. Top Panels: Rapidity dependence. Bottom Panels: Transverse momentum dependence at midrapidity, $\left|y_{0}\right|<0.3$. Upper curves for $m_{n}^{*}>m_{p}^{*}$, lower curves for the opposite splitting $m_{n}^{*}<m_{p}^{*}$. Left: Asystiff. Right: Asysoft.

reached maximum density is not much different in the two cases. The same is observed for the isospin flows analyzed in the following Subsection.

We remark that all the effects discussed before should be also present for the ${ }^{3} \mathrm{H} /{ }^{3} \mathrm{He}$ yield ratios, more easily detected. Particularly interesting is the predicted large increase at high $p_{t}$ in the $m_{n}^{*}<m_{p}^{*}$ choice. Some preliminary FOPI results seem to indicate this trend [26], but more data are needed. It is encouraging that we already see a good Iso - MD dependence of rather inclusive nucleon/cluster emission data. In presence of a good statistics for the detection of high $p_{t}$ particles, a further selection at high azimuthal angles and central rapidities would certainly enhance the sensitivity to the momentum dependence of the Symmetry Potentials. This will introduce the discussion of isospin elliptic flows of the following Subsection. 

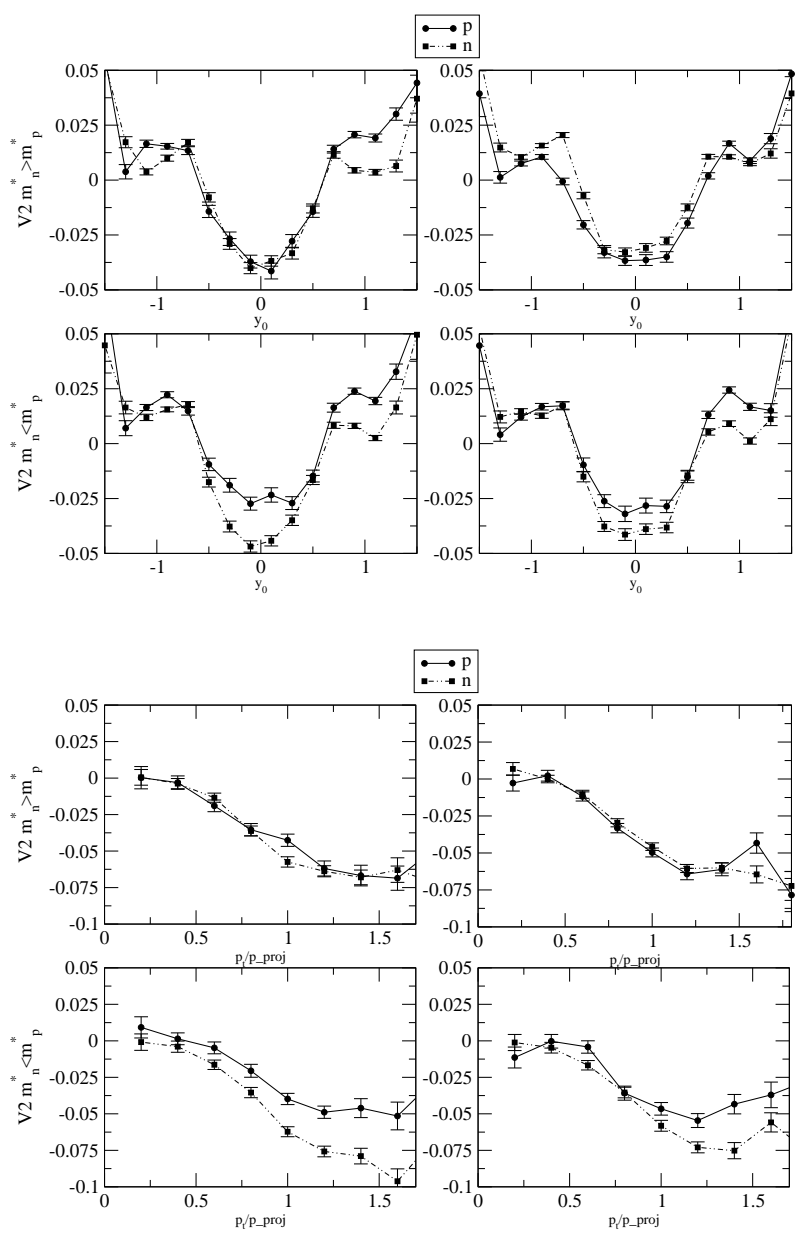

FIG. 9: Same as for Fig 8 for the semi-central reaction $\mathrm{Au}+\mathrm{Au}$ at $600 \mathrm{AMeV}$.

\section{Isospin flows}

Collective flows are very good candidates since they are expected to be very sensitive to the momentum dependence of the mean field, see [1, 27]. The flow observables can be seen respectively as the first and second coefficients from the Fourier expansion of the azimuthal distribution: $\frac{d N}{d \phi}\left(y, p_{t}\right)=N_{0}\left[1+V_{1} \cos (\phi)+2 V_{2} \cos (2 \phi)\right]$, where $p_{t}=\sqrt{p_{x}^{2}+p_{y}^{2}}$ is the transverse momentum and $y$ the rapidity along beam direction. The transverse flow, $V_{1}\left(y, p_{t}\right)=\left\langle\frac{p_{x}}{p_{t}}\right\rangle$, provides information on the anisotropy of nucleon emission on the reaction plane. Very important for the reaction dynamics is the elliptic flow, $V_{2}\left(y, p_{t}\right)=\left\langle\frac{p_{x}^{2}-p_{y}^{2}}{p_{t}^{2}}\right\rangle$. The sign of $V_{2}$ indicates the azimuthal anisotropy of emission: on the reaction plane $\left(V_{2}>0\right)$ or out-of-plane (squeeze - out, $\left.V_{2}<0\right)$ 27].

Isospin effects on collective flows have been studied within the $\operatorname{UrQMD}$ transport model in order to probe the influence of the symmetry repulsion at high densities [28, 29], here we focus the attention on the mass-splitting

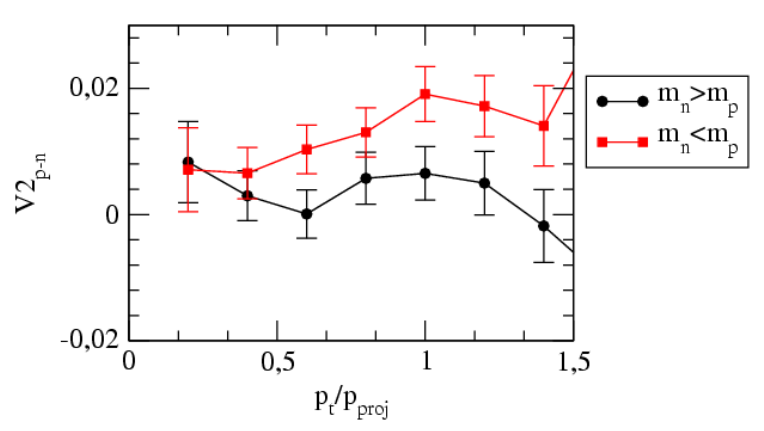

FIG. 10: Transverse momentum dependence of the difference between proton and neutron $V_{2}$ flows, at mid-rapidity, $\left|y_{0}\right|<$ 0.3 , in a semi-central reaction $\mathrm{Au}+\mathrm{Au}$ at $400 \mathrm{AMeV}$. Asystiff choice.

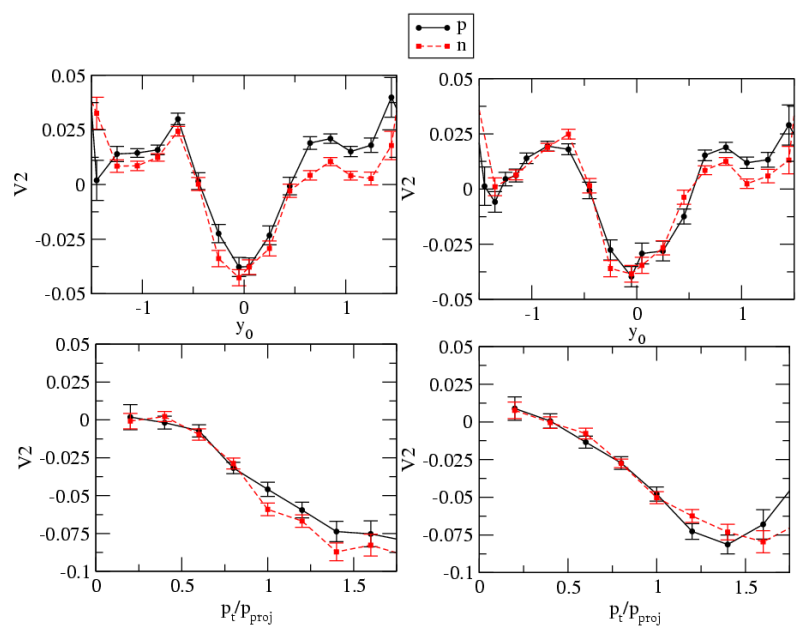

FIG. 11: Proton (thick) and neutron (thin) $V_{2}$ flows in a semi-central reaction $\mathrm{Au}+\mathrm{Au}$ at $400 \mathrm{AMeV}$, for equal (n.p)effective masses. Top Panels: Rapidity dependence. Bottom Panels: Transverse momentum dependence at midrapidity. Left: Asystiff. Right: Asysoft.

contributions. For the same $\mathrm{Au}+\mathrm{Au}$ reactions, in a semicentral selection, we present in Figs. 8, 9(Upper Panels) the rapidity dependence of $(n / p) V_{2}$ for different choices of the Asy-stiffness and effective mass splitting. We observe the relevance of the latter: at mid-rapidity the neutron squeeze-out is much larger in the $m_{n}^{*}<m_{p}^{*}$ case independently of the stiffness of the symmetry term. We note however that in the Asysoft case we see an inversion of the neutron/proton squeeze-out at mid-rapidity for the two effective mass-splittings. Good data seem to be suitable to disentangle $I s o-M D$ potentials.

The mass-splitting effect is large at high $p_{t}$ (Bottom Panels), again in a mid-rapidity selection, as expected for particle emitted from higher density regions. Here the results are also slightly depending on the Asy-stiffness, with large neutron squeeze - out effects in the Asystiff case. 

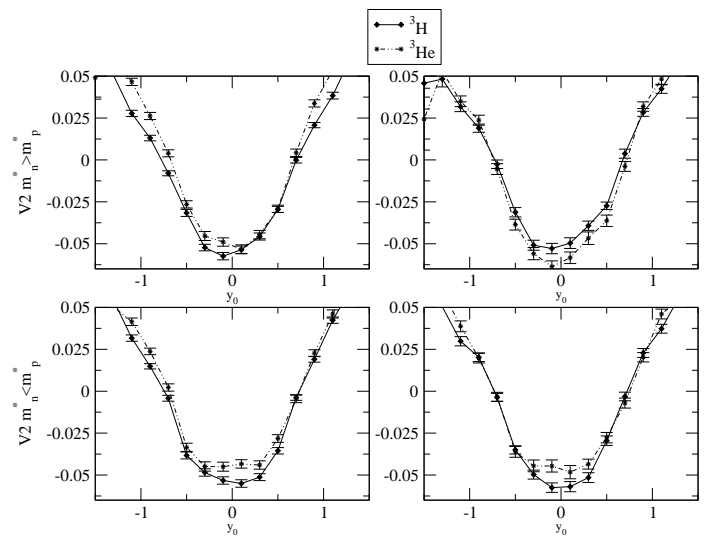

FIG. 12: Rapidity dependence of Triton (thin) and ${ }^{3} \mathrm{He}$ (thick) $V_{2}$ flows in a semi-central reaction $\mathrm{Au}+\mathrm{Au}$ at $400 \mathrm{AMeV}$. Upper curves for $m_{n}^{*}>m_{p}^{*}$, lower curves for the opposite splitting $m_{n}^{*}<m_{p}^{*}$. Left Panels: Asystiff. Right Panels: Asysoft.
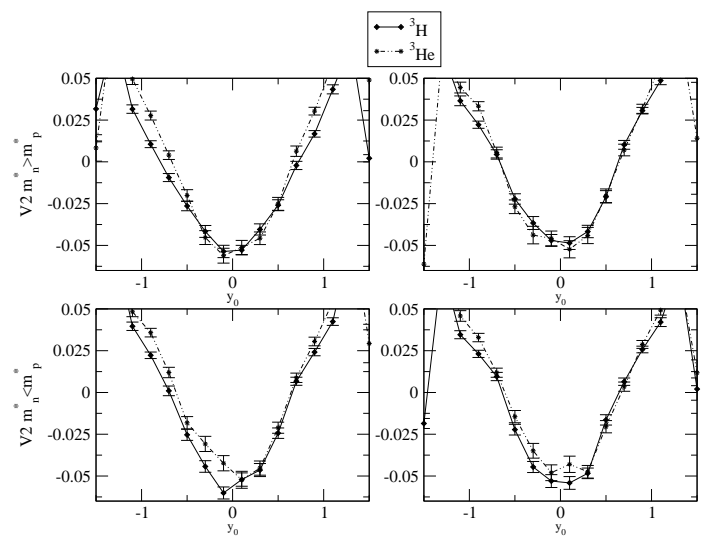

FIG. 13: Same as in Fig 12 for a semi-central reaction $\mathrm{Au}+\mathrm{Au}$ at $600 \mathrm{AMeV}$.

A good sensitive observable seems to be the Difference of $(p / n)$ elliptic flows, $V_{2}(p)-V_{2}(n)$, shown in Fig 10 vs. transverse momentum at mid-rapidity, that is systematically larger in the $m_{n}^{*}<m_{p}^{*}$ case.

In order to have a clear idea of the relevance of the $(n, p)$ mass splitting on the fast nucleon emissions we present in Fig 11 the neutron/proton elliptic flows for semicentral $A u+A u$ collisions at $400 A M e V$ evaluated with the parametrizations giving $m_{n}^{*}=m_{p}^{*}$ for the ${ }^{197} A u$ asymmetry $\beta \simeq 0.2[30]$. Now the isospin effects are only related to the different stiffness of the symmetry term at suprasaturation density. We see that, at variance with the mass-splitting results of Fig 8 , the rapidity distributions (top panels) are not much affected, with a slightly larger neutron squeeze-out in the Asystiff case. Consistently we see some difference in the transverse momentum dependence at mid-rapidity (bottom panels) only at very large $p_{t}$.

Due to the difficulties in measuring neutrons, we have analyzed the isospin sensitivity of light isobar flows, like

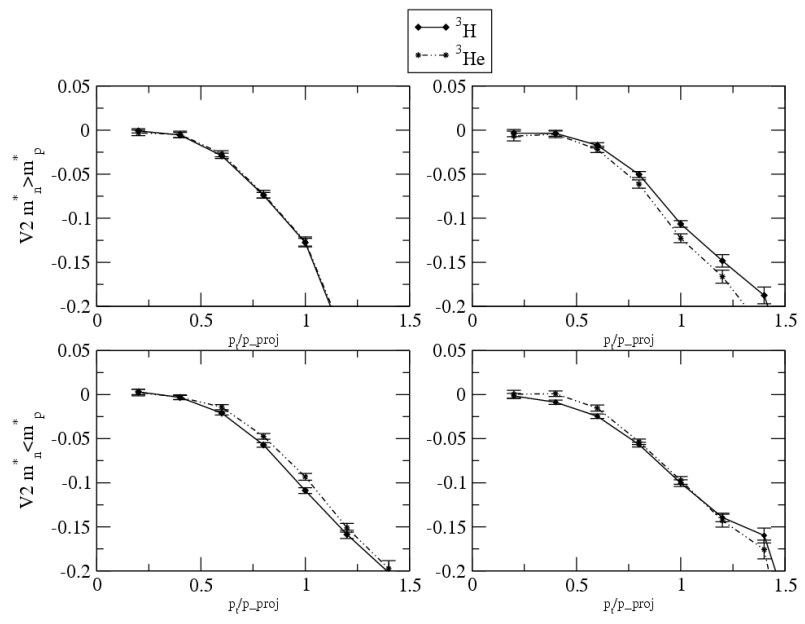

FIG. 14: Transverse momentum dependence at mid-rapidity of ${ }^{3} \mathrm{He}$ (thick) and ${ }^{3} \mathrm{H}$ (thin) $V_{2}$ flows in a semi-central reaction $\mathrm{Au}+\mathrm{Au}$ at $400 \mathrm{AMeV}$. Upper curves for $m_{n}^{*}>m_{p}^{*}$, lower curves for the opposite splitting $m_{n}^{*}<m_{p}^{*}$. Left: Asystiff. Right: Asysoft.

${ }^{3} \mathrm{H}$ vs. ${ }^{3} \mathrm{He}$ and so on. We still see effective mass splitting effects, although slightly reduced, as shown in Figs. 12, 13 for the triton $/{ }^{3} \mathrm{He}$ case.

As in the nucleon elliptic flow, at mid-rapidity the triton squeeze-out is larger in the $m_{n}^{*}<m_{p}^{*}$ case independently of the stiffness of the symmetry term. Again in the Asysoft case we see an inversion of the ${ }^{3} \mathrm{H}$ vs. ${ }^{3} \mathrm{He}$ squeeze-out at mid-rapidity for the two choices of the mass-splitting.

In Fig 14 we show also the the transverse momentum dependence of the ${ }^{3} \mathrm{He},{ }^{3} \mathrm{H}$ elliptic flows at mid-rapidity $\left(\left|y_{0}\right|<0.3\right)$ at $400 \mathrm{AMeV}$. Some mass-splitting effects can be seen at high $p_{t}$. This should be well observed in the flow difference. Unfortunately with the present number of events we cannot present a figure (like the Fig 10 of the $(p, n)$ case) since we have too large error bars for the lack of statistics.

We note that increasing the beam energy from 400 to $600 \mathrm{AMeV}$ the symmetry potential effects are not much changing, as already seen also for the isospin ratios of fast emitted particles discussed in the previous Subsection. In this respect an interesting positive result is coming from preliminary FOPI data on ${ }^{3} \mathrm{H}$ vs. ${ }^{3} \mathrm{He}$ flows, for $\mathrm{Au}-\mathrm{Au}$ collisions at beam energies extended up to $1.5 \mathrm{AGeV}$. The triton $V_{2}$ shows a larger squeeze-out at mid-rapidity (in a relatively high transverse momentum selection) [26], clearly increasing with the beam energy, consistent with the reached higher baryon densities.

\section{CONCLUSION AND PERSPECTIVES}

The paper has been mainly devoted to the study of the effects of the momentum dependence of the inmedium effective nuclear interactions on heavy ion reac- 
tions at intermediate energies. Apart the already known I soscalar effects, like less stopping, less compression and an overall stiffer EoS, we have investigated in detail the Isovector contributions, that give raise to the poorly known neutron-proton effective mass splitting in asymmetric matter. We remind that this point is also of interest for relativistic bosonic models of the nuclear matter [1, 4, 31, 32] and the development of effective Lagranginas for non-perturbative QCD related to the breaking of the chiral symmetry [33 35]. We have shown that the fast nucleon and light ions emissions in heavy ion collisions at intermediate energies are affected by the $(n, p)$ mass splitting. The isospin yield ratios $\left(n / p,{ }^{3} \mathrm{H} /{ }^{3} \mathrm{He}\right)$ and elliptic flows appear more sensitive to the Mass-splitting than to the stiffness of the Symmetry Term at high density, in particular for high transverse momentum selections. This opens the possibility of disentangling the two distinct isospin effects on the reaction dynamics. New more accurate and exclusive data are needed. We like to mention the new measurements that will be performed at SISGSI by the ASYEOS Collaboration [36] and the new experiments planned at RIKEN-Tokyo and CSR-Lanzhou also with unstable, more neutron-rich, beams.

\section{Acknowledgements}

We warmly thank W.Reisdorf, W.Trautmann, P.Danielewicz, W.G.Lynch, Qinfeng Li and P.Russotto for several nice and inspiring discussions.
[1] V. Baran, M. Colonna, V. Greco, M. Di Toro, Phys. Rep. 410 (2005) 335.

[2] B.A. Li, L.W. Chen, C.M. Ko, Phys. Rep. 465 (2008) 113.

[3] M. Di Toro et al., Progr. Part. Nucl. Phys. 62 (2008) 389.

[4] C. Fuchs, H.H. Wolter, Eur. Phys. Jour. A30 (2006) 5.

[5] P. Chomaz, M. Colonna, J. Randrup, Phys. Rep. 389 (2004) 263.

[6] J. Rizzo, P. Chomaz, M. Colonna, Nucl. Phys. 806 (2008) 40.

[7] M. Colonna, M. Di Toro, G. Fabbri, S. Maccarone, Phys. Rev. C57 (1998) 1410.

[8] M. Di Toro, M. Colonna, J. Rizzo, AIP Conf. Proc. 791 (2005) 70-83.

[9] A. Guarnera, M. Colonna, P. Chomaz, Phys. Lett. B373 (1996) 267.

[10] M. Colonna, M. Di Toro, A. Guarnera, S. Maccarone, M. Zielinska-Pfabe, H.H. Wolter, Nucl. Phys. A642 (1998) 449.

[11] M. Colonna, G. Fabbri, M. Di Toro, F. Matera, H.H. Wolter, Nucl. Phys. A742 (2004) 337.

[12] A. Guarnera, TWINGO Code, Ph.D. Thesis, Univ. of Caen 1996

[13] J. Rizzo, Collective and Stochastic Observables in HIC at Intermediate Energies Ph.D. Thesis, Univ. Catania 2006

[14] G.Q. Li, R. Machleidt, Phys. Rev. C48 (1993) 1702; Phys. Rev. C49 (1994) 566.

[15] E. Santini, T. Gaitanos, M. Colonna, M. Di Toro, Nucl. Phys. A756 (2005) 468.

[16] J. Rizzo, M. Colonna, M. Di Toro Phys. Rev. C72 (2005) 064609.

[17] J. Rizzo et al., Nucl. Phys. A806 (2008)79-104.

[18] C. Gale, G.F. Bertsch, S. Das Gupta, Phys. Rev. C35 (1987) 1666

[19] C. Gale, G.M. Welke, M. Prakash, S.J. Lee, S. Das Gupta, Phys. Rev. C41 (1990) 1545.

[20] I. Bombaci et al., Nucl. Phys. A583 (1995) 623.

[21] V. Greco, Master Thesis, Catania Univ. (1997);
V. Greco, A. Guarnera, M. Colonna, M. Di Toro, Phys. Rev. C59 (1999) 810;

Nuovo Cimento A111 (1998) 865.

[22] B.A. Li, C.B. Das, S. Das Gupta, C. Gale, Nucl.Phys. A735 (2004) 563

[23] P. Danielewicz, R. Lacey and W.G. Lynch, Science 298, (2002) 1592.

[24] Valentina Giordano, Master Thesis, Univ. of Catania 2008, and contribution to the Workshop HIC at Low and Intermediate Energies ECT*-Trento, May 2009, on the Web.

[25] A. Andronic, J. Lukasik, W. Reisdorf, W. Trautmann, Eur. Phys. J. A30, (2006) 31.

[26] W. Reisdorf and FOPI Collab., private communication and ECT*-Trento Workshop as in 24], on the Web.

[27] P. Danielewicz, Nucl. Phys. A673 (2000) 375.

[28] Q. Li, Z. Li, S. Soff, M. Bleicher, H. Stoecker, Phys. Rev. C72 (2005) 034613; Q. Li, Z. Li, H. Stoecker Phys. Rev. C73 (2006) 051601(R).

[29] Q. Li et al., J. Phys. G32 (2006) 151.

[30] We remark that, due to the different neutron/proton Fermi momentum in isospin asymmetric matter, this parametrization is different from the one without Isovector contributions to the momentum dependence, i.e. with the choice $z=C / 8$, see Eq. (5).

[31] B. Liu, V. Greco, V. Baran, M. Colonna, M. Di Toro, Phys. Rev. C65 (2002) 045201.

[32] C. Fuchs, Prog. Part. Nucl. Phys. 56 (2006) 1-103.

[33] M. Frank, M. Buballa, M. Oertel, Phys. Lett. B562 (2003) 221.

[34] G.Y. Shao, L. Chang, Y.X. Liu, X.L. Wang, Phys. Rev. D73 (2006) 076003.

[35] S. Plumari, Ph.D. Thesis, Univ. Catania (2009).

[36] R.C. Lemmon, P. Russotto et ASYEOS Collab., Constraining the symmetry energy at suprasaturation densities with measurements of neutron and proton elliptic flows, Exp. proposal accepted at SIS-GSI (2009). 